\title{
Quantitation of human milk fat globule (HMFG1) expression in breast carcinoma and its association with survival
}

\author{
D M PARHAM, W SLIDDERS, A J ROBERTSON \\ From the Department of Pathology, University of Dundee, Ninewells Hospital and Medical School, Scotland
}

SUMMARY Expression of human milk fat globule (HMFG1) in immunohistochemically stained sections of breast carcinomas was assessed subjectively and objectively from 82 women (age range 4196 years) to determine its prognostic importance. No correlation was observed between the degree of staining and prognosis even when the subcellular distribution of antigen expression was assessed. The total absence of staining with HMFG1 was possibly associated with a favourable outcome, although this did not quite achieve significance with the small numbers involved. The Quantimet 970 was used for objective semiautomated measurement of immunohistochemical reactions in paraffin wax sections and was found to produce better resolution and to eliminate subjective error.

In his study of squamous carcinoma of the lip Broders was one of the earliest workers to note the association between the histological differentiation of a tumour and its prognosis.' The degree of differentiation of most tumours is now considered by many pathologists and clinicians to have some prognostic value. Bloom and Richardson applied this hypothesis to breast carcinomas and separated them into three histological grades. $^{2}$ This grading method, which subjectively assesses tubular differentiation, mitotic index, and nuclear characteristics, has been shown to lack interobserver reproducibility and is only moderately predictive of prognosis for grades 1 (low) and 3 (high), with less certainty for grade $2{ }^{3}$ There has been some evidence to suggest that the combination of histological grade and tumour stage provides a better prognostic index. ${ }^{4}$

The recent development of monoclonal antibodies directed against normal constituents of breast duct epithelium ${ }^{5}$ has raised the possibility that expression of these antigens may be related to the degree of cell differentiation and hence prognosis. Present methods of grading antigen expression in paraffin wax sections are subjective and lack precision. This problem may be overcome by image analysis which permits accurate quantitation of immunoreactivity.

In this retrospective study of cases of known survival of breast carcinoma we present our findings on HMFG1 quantitation by standard light microscopical examination and automated image analysis.

Accepted for publication 17 March 1988

\section{Material and methods}

One hundred and twenty six women, mean age 67 years (range 41-96), were selected from the Tayside Cancer Registry. They had been consecutively diagnosed as having primary infiltrating ductal adenocarcinoma in Maryfield Hospital and Dundee Royal Infirmary between 1972 and 1973 . Surgical treatment consisted of simple mastectomy and radiotherapy ( $\mathrm{n}$ $=104)$, simple mastectomy $(n=11)$, wedge resection with radiotherapy $(n=8)$, or only wedge resection ( $n$ $=3$ ). Information regarding menstrual status of the patients was not available. Follow up for the purpose of this study stopped in June 1987 at which time 23 patients remained alive. For assessment of human milk fat globule (HMFG1) staining, 44 cases had to be excluded from the study due to loss of the original tissue from files $(n=9)$, extensive tumour necrosis ( $=9$ ), or because the amount of remaining tumour tissue was indequate $(n=26)$.

Formalin fixed paraffin wax sections $5 \mu$ m thick were used. These were stained in a single batch with HMFGI (Unipath) by the avidin-biotin peroxidase label method (Vector Laboratories). ${ }^{6}$ The binding resulted in a brown reaction product following oxidation of diaminobenzidine. Sections were counterstained with alum haematoxylin.

The sections were examined by light microscopy by two observers without knowledge of the patients' survival. Where variance on the assessment of particular features occurred, a concensus decision was reached by review and discussion. The histological 
grade was determined on the original haematoxylin and eosin stained sections by the method devised by Bloom and Richardson ${ }^{2}$ and described in detail by Elston. $^{7}$

\section{ASSESSMENT OF HMFGI IMMUNOREACTIVITY}

Light microscopy Visual assessment of tumour immunoreactivity was carried out by the method outlined by Wilkinson et al..$^{8}$ The distribution of staining was assessed at low power $(\times 40)$ and evaluated as follows: (a) no staining throughout the section; (b) non-uniform staining with some areas not staining; (c) uniform staining with all areas staining. HMFGl may be located within the tumour cell cytoplasm, on the cell membrane, or extracellularly. For each of these locations the following three features were examined:

\section{Distribution of staining}

The tumours were assessed and scored as follows: (a) focal staining confined to a single HPF (high power field $\times 400$ ), score 1 point; (b) non-uniform stainingat least two HPF staining but separated by nonstaining areas, score 2 points; (c) uniform stainingstaining at all HPF, score 3 points.

2 Proportion of cells staining

This was performed in a area where the staining in the compartment in question was most prominent at high power $(\times 400)$ over five fields. A score of 1 point was allocated when $<50 \%$ of cells stained, 2 points when $50-80 \%$ stained, and 3 when $>80 \%$ stained. For the extracellular staining category the number of deposits were counted per HPF: $<5$ scored 1 point, 5-20 scored 2 points, and $>20,3$ points.

\section{Staining intensity}

The staining intensity was graded as low (1 point), moderate ( 2 points), and high ( 3 points).

For each location an accumulative score was obtained. This resulted in three groups: group $1 \leqslant 4$ points, group $2,5-6$ points, and group $3, \geqslant 7$ points.

Image analysis The proportion of the neoplasm occupied by malignant cells was determined by the method previously described by Parham et al using sections stained immunohistochemically. To evaluate the Quantimet 970 performance the immunoreactivity of the sections was initially assessed visually (at $\times \mathbf{4 0}$ and $\times 400$ ) as follows: (i) no positive staining; (ii) less than $20 \%$ of tumour cells staining; (iii) $20-50 \%$ of tumour cells staining; (iv) $51-70 \%$ of tumour cells staining; (v) greater than $70 \%$ of tumour cells staining.

Image analysis was performed on the Quantimet 970 (Cambridge Instruments Ltd). The system comprises a central processor with display and computer. The analysis is based on the television scanner converting the histological image into 500000 pixels. The Quantimet can distinguish 256 grey levels or grades of density between white and black, and the grey level of each picture point can be recorded. Features at or above a preset threshold can be quantified by the central processor, with visual control by the video monitor.

Three grey level thresholds were used to detect HMFG1 staining, malignant cells, and stroma, respectively. The area of each microscopic field (total magnification $\times 80$ ) occupied by each component was determined. Electronic editing was used to eliminate section and staining artefacts. The area occupied by HMFG1 was expressed as a percentage of the area occupied by malignant cells (labelled percentage area) and similarly the area'occupied by malignant cells expressed as a percentage of the total neoplasm areathat is, malignant cells plus stroma (malignant cell percentage area). Due to the non-uniform distribution of malignant cells in a neoplasm, consecutive nonoverlapping fields were evaluated across a section from the periphery to the centre and back. Depending on size of neoplasm this was repeated until a total of 20 fields had been examined and the average result calculated. A preliminary trial showed that accurate results for each of the measurements could be obtained following 10 consecutive fields (results not shown).

Analysis of survival data was carried out using life tables and the log rank test as described by Peto et al..$^{10}$

\section{Results}

No difference in the survival pattern was observed between the 82 cases assessed immunohistochemically and those excluded due to insufficient material (log rank test: $\left.\chi^{2}=0 \cdot 118,1=\mathrm{df}, \mathrm{p}>0.7\right)$, indicating that no bias of the data would result from the excluded cases. A previous study has indicated that extensive tumour necrosis was associated with a particularly poor prognosis.

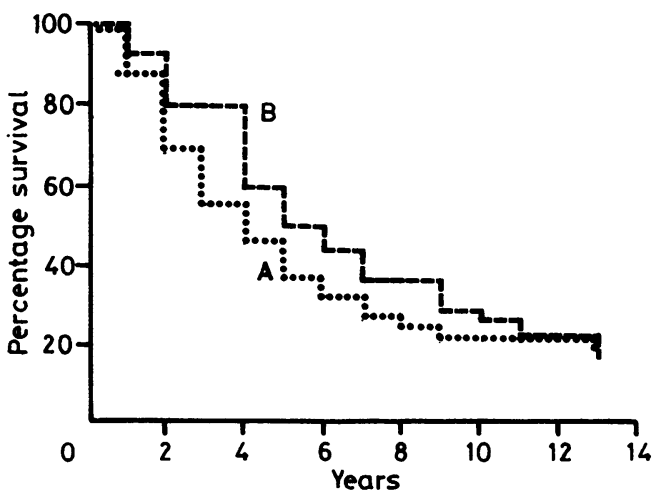

Fig 1 Life table showing survival patterns between carcinomas exhibiting non-uniform ( $a$ ) and uniform (b) HMFGI staining. 
STAINING DISTRIBUTION WITHIN A NEOPLASM

Three tumours did not show any immunoreactivity with HMFGl. At the end of follow up two of these patients were alive and one had died after 175 months. This result did not quite achieve significance when compared with those tumours that did stain (log rank test: $\left.\chi^{2}=3 \cdot 35,1=\mathrm{df}, \mathrm{p}>0 \cdot 1\right)$. Fig 1 shows that there was no significant difference in the survival curves between those tumours exhibiting non-uniform (a) and uniform (b) staining patterns (log rank test: $\chi^{2}=$ $0.98,1=\mathrm{df}, \mathrm{p}>0.3)$.

\section{SEMIQUANTITATIVE ASSESSMENT OF STAINING}

Seventy eight of the 82 tumours exhibited evidence of cytoplasmic staining, 68 membranous staining, and 72 extracellular staining. Fig 2 shows the survival curves for those with low $(n=34)$, moderate $(n=34)$, and high $(n=14)$ levels of extracellular staining. Life table analysis $\left(\log\right.$ rank test $\chi^{2}=1.41,2=\mathrm{df}, \mathrm{p} \simeq 0.5$ ) showed no significant difference between the three groups. Similar results were obtained for cytoplasmic and membranous staining (data not shown). No association was found between the grade of staining in each of the compartments and either histological grade or patient age.

\section{IMAGE ANALYSIS}

The association between the visual quantitation of the proportion of a neoplasm occupied by malignant cells and the Quantimet assessment is shown in fig 3. The Quantimet tends to underestimate slightly the proportion of a neoplasm occupied by malignant cells compared with the visual assessment, although the correlation between the results is excellent. Similarly, the correlation between the staining for HMFG1 when assessed visually and by the Quantimet is shown in fig 4. Considerable overlap between the groups assessed visually and the Quantimet assessment of staining was

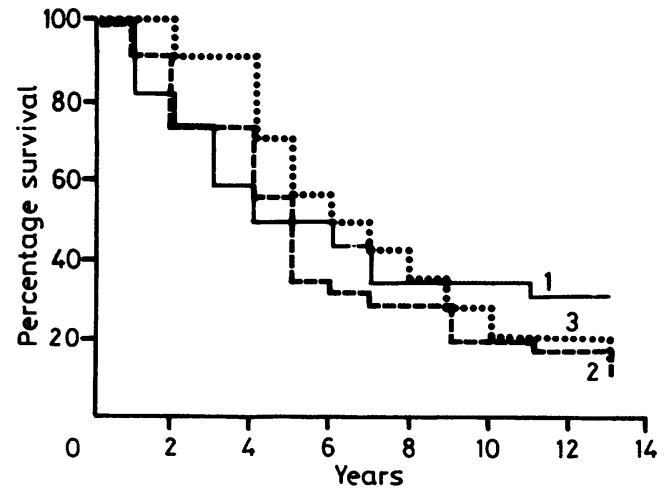

Fig 2 Life table showing survival patterns for breast carcinomas with low (1), moderate (2), and high (3) levels of extracellular HMFG1 staining.

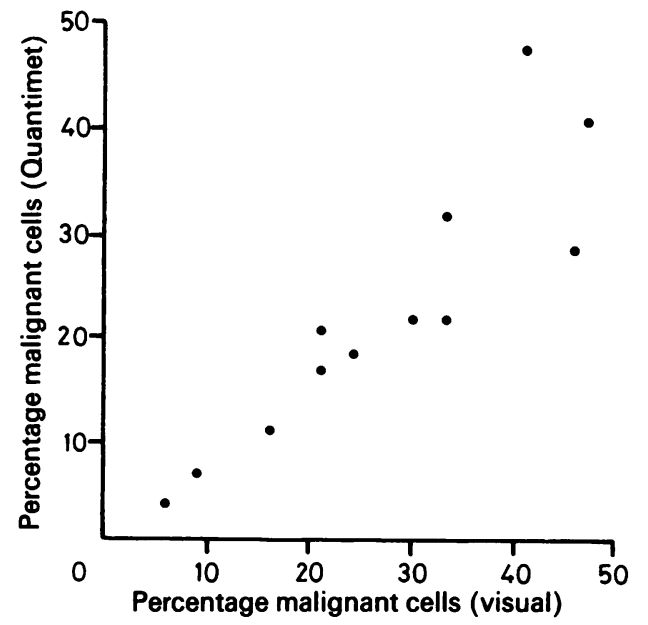

Fig 3 Relation between visual assessment of percentage of tumour area occupied by malignant cells and the Quantimet measurement $(Y=0.85 X-0.46, R=0.89)$.

seen. The labelled percentage area in the 82 cases examined ranged from 0.001 to $18.1 \%$ (mean $3.69 \%$ ). Examination of the 25th, 50th, 75th, and 100th percentiles showed that 20 patients had a percentage of labelled tumour cell area of less than $0.37 \%$ (group 1), 21 patients had values between $0.38 \%$ and $1.56 \%$ (group 2), 21 patients had values between $1.79 \%$ and $5.76 \%$ (group 3), and 20 patients a percentage of labelled tumour cell area of greater than $5.8 \%$. No significant difference in the survival curves for these four groups was observed (log rank test $\chi^{2}=3 \cdot 50$, $3=\mathrm{df}, \mathrm{p}>0 \cdot 3$ ). Exclusion of the three non-staining

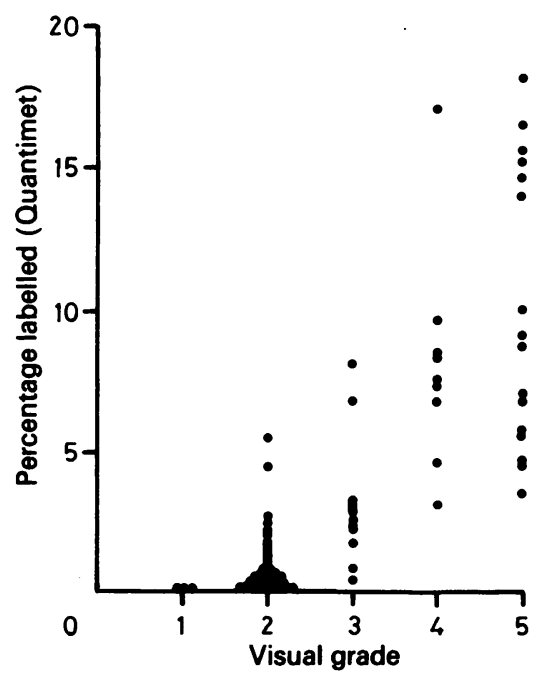

Fig 4 Relation between visual quantitation of HMFGI staining in 82 breast carcinomas and Quantimet analysis. 


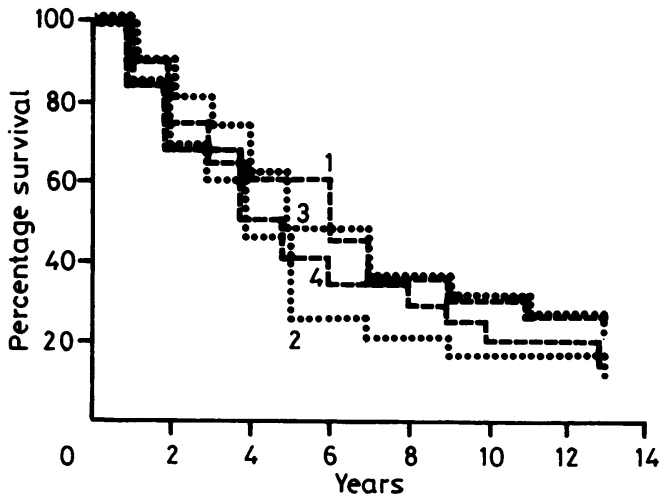

Fig 5 Survival curves for staining groups as assessed by Quantimet analysis (group $1 \leqslant 0.43 \%$, group $20.44-$ $\leqslant 1 \cdot 8 \%$, group $31 \cdot 85-5 \cdot 8 \%$, group $4>6 \cdot 7 \%$ ).

tumours and separation of the remaining 79 cases into four groups $(\leqslant 0.43 \%, 20$ cases; $0.44-\leqslant 1.8 \%, 20$ cases; $1 \cdot 8-5 \cdot 8 \%, 20$ cases, $>6.7 \%, 19$ cases) resulted in closer approximation of the survival curves (fig $5, \log$ rank test: $\chi^{2}=2 \cdot 36,3=\mathrm{df}, \mathrm{p} \simeq 0.5$ ).

\section{Discussion}

The overall HMFGl staining characteristics of the tumours used in this study are similar to those observed by Wilkinson et al. ${ }^{8}$ Their findings, however, showed that an absence of antigen expression was associated with a poor prognosis. In contrast, our findings showed that the three tumours with no staining had an apparently better than average prognosis, although this did not quite achieve significance, probably due to the small numbers studied. The smaller proportion of tumours not reacting with HMFGl in our study may in part reflect the greater sensitivity of the avidin-biotin method of staining. ${ }^{6}$ For those tumours that did stain, the indices derived for each compartment showed no correlation with survival. These results are also at variance with the findings of Wilkinson et al, ${ }^{8}$ who found that extracellular deposition was associated with a favourable prognosis. This discrepancy may relate to differences in the length of follow up after diagnosis in the women studied. In the study by Wilkinson et al the mean follow up was only 36 months, and their findings related to the relapse free period rather than that of postoperative survival as in our study. Another factor, which may explain the difference noted in the association between extracellular staining and prognosis, is the complex nature of the grading system. For a grading system to be of value it must be simple and reproducible. In particular, when assessing extracellular deposits, we noted that sometimes antibody-antigen reaction production was present in areas where the pattern of fibrosis suggested recent tumour cell necrosis. In such cases this would not represent true extracellular secretion. A simpler scoring system devised by Berry et $a l^{11}$ in which grading was based on the site of strongest staining and the extent of intracellular staining also found HMFG1 to be of no prognostic value. Ellis et al ${ }^{1213}$ using another monoclonal antibody (NCRC 11) raised to human milk fat globule membrane, and which exhibits a similar staining pattern, have recently shown a positive relation between the degree of staining and survival using a subjective method of quantitation.

The subjective nature of visually quantifying immunoreactivity is prone to considerable inter- and intraobserver error, although this can be reduced by forming fewer staining categories but at the risk of losing prognostic information. For this reason objective quantitation of HMFG1 staining was carried out by image analysis, HMFG1 staining being expressed as a percentage of the area occupied by tumour cells, as the proportion of malignant cells in different neoplasms has been shown to vary widely. ${ }^{914}$ Extracellular deposits contribute negligibly to the overall assessment. Our study has shown the considerable degree of overlap that occurs between groups of tumours when visually quantified compared with the results obtained by Quantimet image analysis. The greater precision of the Quantimet in measuring the area occupied by antigen-antibody product permits better resolution and eliminates subjective error.

The results obtained, however, show no association with survival suggesting that quantitation of HMFG1 expression is of little prognostic value. The technique is unable to distinguish antigen expression at different sites within a neoplasm, although the semiquantitative results suggest that this is not important. The results of this study do not totally negate the possibility that HMFGl expression is related to prognosis as the analysis did not take into account the intensity of each reaction product deposit. It may be possible to achieve more detailed comparison of sections by determining the optical density of reaction product deposits present. Millar and Williams ${ }^{15}$ have shown that high concentrations of antigen may actually diminish the density of staining. To guard against this and for comparison of different staining batches a step wedge method $^{15}$ of calibration ought to be utilised.

We thank Mr G Coghill and Mr R Fawkes for their technical assistance and Professor J S Beck for his encouragement.

\section{References}

1 Broders AC. Squamous epithelioma of the lip: A study of five hundred and thirty seven cases. JAMA 1920;74:656-64. 
2 Bloom HJG, Richardson WW. Histological grading and prognosis in breast cancer. Br J Cancer 1957;11:359-77.

3 Rich MA, Brennan MJ. The breast cancer prognostic program: a study of the metastatic process, In: Brennan MJ, McGrath CM, Rich MA, eds. Breast cancer: new concepts in etiology and control. New York: Academic Press, 1980:29-51.

4 Haybittle JL, Blamey RW, Elston CW, et al. A prognostic index in primary breast cancer. Br J Cancer 1982;45:361-6.

5 Taylor-Papadimitriou J, Peterson JA, Arklie J, et al. Monoclonal antibodies to epithelium-specific components of the human milk-fat globule membrane: production and reaction with cells in culture. Int J Cancer 1981;28:17-21.

6 Coggi G, Dell'Orto P, Viale G. Avidin-biotin methods. In: Polak JM, Van Noorden S eds. Immunocytochemistry: modern methods and applications. Bristol: John Wright \& Sons Ltd, 1986:54-70.

7 Elston CW. Assessment of histological grade in human breast cancer. In: Blamey RW, ed. Seminar in breast cancer. London: Update Publications, 1984: 47-50.

8 Wilkinson MJS, Howell A, Harris M, et al. The prognostic significance of two epithelial membrane antigens expressed by human mammary carcinomas. Int J Cancer 1984;33:299-304.

9 Parham DM, Brown RA, Robertson AJ. Morphometric analysis of breast carcinoma: relationship to survival. J Clin Pathol 1988:41:173-7.
10 Peto R, Pike MC, Armitage P, et al. Design and analysis of randomised clinical trials requiring prolonged observations of each patient. Br J Cancer 1977;35:1-39.

11 Berry N, Jones DB, Smallwood J, Taylor I, Kirkham N, TaylorPapadimitriou J. The prognostic value of the monoclonal antibodies HMFG1 and HMFG2 in breast cancer. $\mathrm{Br} J$ Cancer 1985;51:179-86.

12 Ellis IO, Robins RA, Elston CW, Blamey RW, Ferry B, Baldwin RW. A Monoclonal Antibody, NCRC11, raised to human breast carcinoma. 1 Production and immunohistological characterization. Histopathol 1984;8:501-16.

13 Ellis IO, Hinton CP, Macnay J, et al. Immunocytochemical staining of breast carcinoma with the monoclonal antibody NCRC11: A new prognostic indicator. $\mathrm{Br}$ Med J 1985;290: 881-3.

14 Underwood JCE. A morphometric analysis of human breast carcinoma. Br J Cancer 1972;26:234-7.

15 Miller DA, William ED. A step wedge standard for the quantitation of immunoperoxidase technique. Histochem $J$ 1982;14:609-20.

Requests for reprints to: Dr D M Parham, Department of Pathology, Ninewells Hospital and Medical School, Dundee, Scotland, DD1 9SY. 